Ann. Zootech., 1973, 22 (3), 333-336.

NOTE TECHNIQUE

\title{
MÉTHODE DE DÉTECTION DE L'CESTRUS CHEZ LA JUMENT EN LIBERTÉ
}

\author{
B. ARChambeaud ( $\left.{ }^{(}\right)$, M. JUSSiAUX*, E. PALMER et J.-P. SigNoRET \\ Station de Physiologie de la Reproduction, \\ Centre de Recherches de Tours, I. N. R. A., \\ Domaine de l'Orfyasière, \\ Nonzilly, B. P. 1, 37380 Monnaie \\ * Station expérimentale des Haras nationaux, La Valade, \\ 19 Chamberet
}

La détection des chaleurs chez la Jument s'effectue habituellement dans les élevages par la présentation quotidienne à un étalon "souffleur ». Le développement de méthodes d'élevage en plein air permanent rend nécessaire d'autres méthodes adaptées à ces conditions nouvelles. C'est pourquoi nous avons cherché à mettre au point une technique comportant le marquage des femelles par un étalon intact ou vasectomisé restant en permanence avec elles.

L'étude de ce problème a déjà été abordé dans les autres espèces : chez les bovins, le badigeonnage journalier du sternum et de la face interne des membres antérieurs au moyen d'une graisse colorée a été utilisé avec succès dans ce but (Rollinson, 1963. Mills, Turner et VinVENT, 1969).

Chez les ovins, les zootechniciens australiens ont proposé une alternative qui évite les inconvénients de l'application journalière de peinture épaisse ou de graisse : un bloc de paraffine colorée est maintenu en place sur le sternum du mâle par un harnais. La dureté de la paraffine est variable selon la température : trois qualités sont proposées pour temps chaud (au-dessus de $29^{\circ} \mathrm{C}$ ), tempéré ( $\mathrm{I} 5$ à $29^{\circ} \mathrm{C}$ ) et froid (au-dessous de $\mathrm{I} 5^{\circ} \mathrm{C}$ ). Les femelles réceptives sont marquées ainsi d'un trait coloré qui persiste plusieurs jours.

L'observation de l'accouplement chez le Cheval laisse supposer que le contact au niveau de l'interars pourrait être suffisant pour marquer la femelle lorsqu'elle accepte le chevauchement.

Un système de harnais a donc été mis au point pour maintenir à cet emplacement (fig. I) un crayon analogue au modèle utilisé chez les ovins. Équipé d'un tel harnais, un étalon de 5 ans de petite taille et sans origine connue, rendu stérile par vasectomie, a été placé du 4 avril au I6 juin I972 dans un troupeau comprenant 8 juments "Selle Français " de 4 ans et I jument anglo-arabe de 9 ans gestante qui a mis bas en mai 1972. Les détails des marques laissées par le crayon ont été notés tandis que les activités sexuelles étaient observées journellement pendant au moins 3 heures.

(1) Stagiaire E. N. I. T. A. Dijon. 


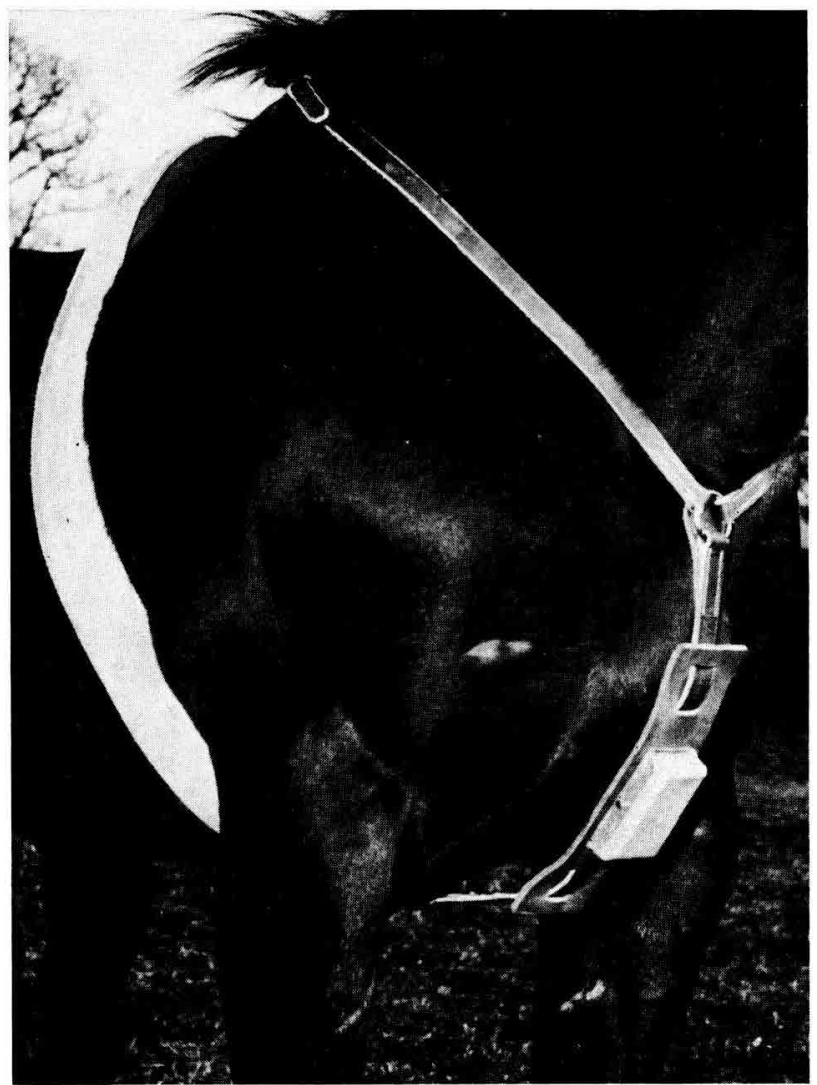

Fig. I. - Harnais marqueur en place sur un étalon

I.e support đu bloc de paraffine colorée normalement maintenu dans l'interars a été déplacé vers l'avant pour qu'il apparaisse nettement sur la photographie

\section{RÉSULTATS}

Les 9 juments expérimentales ont présenté des cycles cestriens réguliers pendant toute la durée des observations.

Le nombre total des jours de chaleur - réceptivité sexuelle à l'étalon - a été de 124 pour l'ensemble des femelles. Un total de 372 marques a été enregistré ; le nombre moyen de marques par jument et par jour de chaleur étant de 2,9 avec une variation dé o à 9 pour un même animal. Divers facteurs influent sur la netteté des traces laissées par le crayon : la coloration de la robe, la longueur et la propreté du poil, etc. Toutes les marques constatées ont été faites sur la croupe de la jument.

Au cours des observations directes des animaux, I 44 activités de monte ont été observées sur des juments en chaleur; il en est résulté 94 marquages. Le détail de ces observations est présenté dans le tableau $\mathbf{I}$.

Ainsi, les deux tiers des activités de monte aboutissent à un marquage de la femelle et près de quatre accouplements sur cinq. 


\section{TABLEAU I}

Marques relevées sur des juments en fonction de l'activité sexuelle d'un étalon équipé d'un harnais marqueur

\begin{tabular}{|c|c|c|c|}
\hline Activité de l'étalon & $\begin{array}{l}\text { Nombre } \\
\text { d'activités } \\
\text { observées }\end{array}$ & $\begin{array}{c}\text { Nombre } \\
\text { de marques } \\
\text { observés }\end{array}$ & $\begin{array}{c}\text { Taux } \\
\text { de marquage } \\
\left(y_{0}\right)\end{array}$ \\
\hline MIontes sans accouplement $\ldots . .$. & 67 & $3^{\prime}+$ & 50,7 \\
\hline Accouplements ............ & 77 & (i) & 78 \\
\hline Activités de montes totales...... & 144 & 94 & 65,3 \\
\hline
\end{tabular}

Dans les conditions de notre observation, le mâle a en moyenne marqué près de trois fois par jour chaque jument en chaleur. Ceci correspondrait à unc moyenne supérieure à 3 accouplements; le pourcentage de chances pour qu'une jument en chaleur soit ainsi identifiée est donc au vu de ces chiffres très élevé. Les observations pratiques ont confirmé cette conclusion théorique : sur l'ensemble des cas de réceptivité sexuelle observés ( 124 jours $\times$ jument), il n'en a été enregistré que quatre où une femelle subissant des activités de monte par l'étalon n'a pas été marquée au cours de la journée. Or, la quasi-totalité des juments ont des chaleurs dont la durée dépasse 3 jours (moyenne: 6-7 jours; Jussiaux et Taillandier, I97 I Nishikawa, I959). Dans le cas de nos observations, 92,9 p. Ioo des chaleurs avaient une durée comprise entre 3 et 8 jours, les 7 , I p. Ioo restant étant supérieures ou égales à 9 jours. La détection d'une période de réceptivité est donc obtenue avec une très bonne précision par le marquage lors de l'accouplement avec un étalon vasectomisé. En aucun cas un essai de chevauchement d'une jument non réceptive n'a permis de marquage, non plus que les montes mal orientées sur une femelle en chaleur (par le côté). Il est possible que le comportement de l'étalon utilisé et la taille du groupe de juments puissent faire varier quelque peu la qualité des résultats et il est nécessaire de les confirmer dans d'autres conditions. Toutefois, cette technique nous semble prometteuse : elle permettrait de : suivre de plus près la reproduction en liberté par la connaissance des dates d'accouplements : estimation des dates de poulinage, détection des juments stériles présentant, soit un anœstrus - complet, soit des chaleurs répétées. Dans le cas de la monte en main, elle pourrait être utilisée pour la détection de l'œstrus avec un mâle vasectomisé.

Rę̧u pour publication en avril 1973.

\section{SUMMARY}

\section{A TECHNIQUE FOR THE IDENTIFICATION OF MATING IN FREEIY RUNNING MARES}

A technique for the identification of mating of mares has been tested in a herd of 9 cyclic mares running freely with a vasectomized male fitted with a stallion harness and crayon.

Sixty-five percent (94/144) of observed mounts were accompanied by corresponding raddle marks. Each mare was marked 2.9 times per day of heat so that in only 3.1 p. Ioo (4/I24) of occa:sions did a mare fail to be marked during a complete day.

The method seems promising for the detection of mating in the mare. 


\section{RÉFÉRENCES BIBLIOGRAPHIQUES}

Jussiaux M., Taillandier F., I97I. Bilan de trois années d'expérimentation à la jumenterie de la rivière. Bull. Tech. Inform. Minist. A gric. 260, I-I2.

Mills A. C., Turner J. W., Vinvent C. K, I969. Method of cestral detection in crossbred cows. J. Anim. Sci. 28, I46 (Abstr.).

Nishikawa Y., I959. Studies on reproduction in horses. Japan. Racing. Ass. éd., Shiba Tamuracho Minatoku, J ckyo, Japan.

Rollinson D. H. L., I963. Reproductive habits of indigenous cattle to artificial insemination in Uganda. J. A gric. Camb. 60, $279-284$. 